\title{
Acknowledgement of Referees
}

The Editors express their gratitude to the following individuals, who served as referees of manuscripts for the Internal Medicine in 2010.

ABe Masahiro

ABE Tadashi

ADACHI Kyoichi

ADACHI Mitsuru

Agematsu Kazunaga

AIBA Takeshi

AIZAWA Hisamichi

Aizawa Yoshifusa

AKAMATSU Naoki

AkAMATSU Taiji

AKASAKA Takashi

AkAshiBA Tsuneto

AKITA Hirotoshi

AKIYAMA Kazuo

AKIYAMA Yasushi

AMANO Koichi

AMANO Yuji

AMESHIMA Shingo

AMINO Nobuyuki

ANDo Katsuyuki

ANDO Minoru

ANDO Yukio

ANDOH Akira

Aoki Nobuki

AOKI Sadao

AOKI Yosuke

AOYAGI Kunihiko

AOYAGI Yutaka

AOY AMA Hidefumi

Arai Ayako

ARAI Masahiro

ARAI Masashi

ARAKAWA Tetsuo

ARAKI Nobuo

ARAKI Tsutomu

ARASE Yasuji

ARIMA Nobuyoshi

ARIMOTO Takanori

ARIMURA Kimiyoshi

ARIMURA Yoshihiro

ARIYOSHI Koya

ASAHINA Yasuhiro

ASAKURA Hidesaku

ASANO F

AsANO Koichiro

ASANO Naoko

Ashino Yugo

Ashizawa Kazuto

Aso Yoshimasa

ASOU Norio

ATARASHI Hirotsugu

Atsumi Tatsuya

Atsumi Yoshihito

AzUma Arata

Azuma Takeshi

BABA Eishi

BABA Hideo

BABAzono Tetsuya

BANDo Masashi
BANDO Yukihiro

Chayama Kazuaki

ChiBA Atsuro

ChIBA Hirofumi

CHIBA Toshimi

CHIDA Kingo

ChIGUSA Yuichi

ChIn Kazuo

Chinushi Masaomi

DAIDA Hiroyuki

DAN Kazuo

DOBASHI Kunio

DoI Naofumi

DoI Ryuichiro

EBINA Masahito

EGUCHI Yuichiro

EMOTO Naoya

ENDO Hirahito

FujII Eitaro

FUJII Takeshi

FUJIKI Akira

FUJIMORI Shunji

FUJIMORI Takahiro

FUJIMORI Yoshihiro

FujIMOTo Keisaku

Fujimoto Kenichi

FujIMOTo Shinichi

Fujimoto Shinpei

FUjIMURA Masaki

Fujishima Seitaro

FujISHIRO Mitsuhiro

FujITA Hiroyuki

FujiTa Jiro

FujıTA Masaki

Fujita Masashi

Fujita Yoshiro

FujIUCHI Satoru

FUJIWARA Kazuo

Fujiyama Yoshihide

FUKAGAwa Masafumi

FUKAO Atsushi

FUKAO Toshiyuki

FUKATSU Atsushi

FuKAzAwa Ryuji

FUKUDA Izumi

FUKUDA Michio

FUKUDO Shin

FUKUI Michiaki

Funumoto Seiji

Fukumoto Yoshihiro

FuKUOKa Kazuya

FukUtake Toshio

FUKUYAMA Hidenao

FunAKOSHI Akihiro

FunAzAKI Toshikazu

Furuichi Kengo
Furukawa Kinya

FURUKAwA Tatsuo

Furuse Junji

FURUSU Akira

FURUSYO Norihiro

FURUTA Takahisa

FURUYA Ryuichi

GEJYo Fumitake

GEMma Akihiko

GoMI Harumi

GoNo Takahisa

GoTo Hajime

GoTOH Jun

GOYA Masahiko

Hisamatsu Tadakazu

HACHISUKA Kenji

HAGIWARA Koichi

HAMADA Junichi

HAMAGUCHI Motohiro

HAMAGUCHI Tomoya

HAMANAKA Ichiro

HAMANO Hideaki

HANADA Hiroyuki

HANAFUSA Toshiaki

HANAJIMA Ritsuko

HANAOKA Masayuki

HANAZAKI Kazuhiro

HANDA Hiroshi

HARA Masumi

HARA Taiga

HARA Toshiro

HARADA Kenichi

HARADA Masaru

HARADA Takashi

HARIGAE Hideo

HARIGAI Masayoshi

HASEGAWA Hitoshi

HASEGAWA M

HASEGAWA Masato

HASEGAWA Naoki

HASEGAWA Osamu

HASEGAWA Yasuhiro

HASHIGUCHI Teruto

HASHIMOTO Etsuko

HASHIMOTO Kozo

HASHIMOTO Shu

HASHINO Satoshi

HASSAN Mohamed

HATA Hiroyuki

HAYASHI Akito

HAYASHI Jun

HAYASHI Matsuhiko

HAYASHI Michio

HAYASHI Shinichiro

HAYASHI Toshio

HIDAKA Hisashi

Higa Futoshi

HIGAKI Jitsuo
HigashiHARA Masaaki

Hige Shuhei

HigUCHI Kazuhide

HiNo Masayuki

HiNODA Yuji

HIRAI Toyohiro

HIRAKATA Yoichi

HiRAMATSU Kazufumi

HIRAMATSU Naoki

HiRANO Kenji

HIRANO Teruyuki

HIRANO Tsutomu

HIRAO Kenzo

HiRAOKA Atsushi

HiRATa Kazuto

HiRATA Kenichi

HIRATA Koichi

HIRAYAma Atsushi

HIROHATA Shunsei

HIROKAwa Makoto

HIROMURA Keiju

HIRONO Osamu

HiRose Hiroshi

HIROSE Takashi

HISAI Hiroyuki

Hisanaga Kinya

HISATOME Ichiro

Hishinuma Akira

Homma Sakae

HoNDA Miwako

HoNDA Takayuki

HoRI Akihiro

Hori Seiji

HoRI Toshiyuki

HORIE Minoru

HoRIE Shigeo

HoRII Manabu

HORIIKE Norio

HORIKAWA Yukio

HORIO M

HoRITA Tetsuya

HORIUCHI Hisanori

HosHi Akihiko

HosHino Haruhiko

Hoshino Tomoaki

Hoshino Yoichi

Hosono Osamu

Hsu Bang-Gee

HYODO Ichinosuke

IBAYASHI Setsuro

ICHIDA Takafumi

ICHIWATA Toshio

IDA Hiroaki

IDE Toshimitsu

IDEISHI Munehito

IDO Akio

IEKo Masahiro

IEMURA Motofumi

IIDA Masato 
IIDA Shinsuke

IIJIMA Kazumoto

IINO Yasuhiko

IINUMA Yoshitsugu

IITAKA Makoto

IIzUKA Katsumi

IIzUKA Masahiro

IIzUKA Takahiro

IKARI Yuuji

IKEDA Akio

IKEDA Kenji

IKEDA Kozo

IKeDA Kozue

IKEDA Norihiko

IKEDA Shu-ichi

IKEDA Tadashi

IKEMATSU Hideyuki

IKUSAKA Masatomi

IKUYAMA Shoichiro

IMAGAWA Akihisa

IMAI Enyu

IMAI $\mathrm{H}$.

IMAI Tsuneo

IMAMURA Masahiro

IMANISHI Yasuo

IMAZEKI Fumio

INABA Masaaki

INAMORI Masahiko

INASE Naohiko

INOKUCHI Kouichi

INOKUMA Shigeko

INOMATA Takayuki

INOUE Akira

INOUE Daisuke

INOUE Fumitaka

INOUE Hiromasa

INOUE Hiroshi

INOUE Ikuo

INoue Kazuaki

InOUE Tatsuhide

InOUE Toru

INOUE Yoshikazu

INOUE Yuichi

INUI Akio

InUI Kazuo

INUKAI Kouichi

INUKAI Toshihiko

INUZUKA Takashi

IRISAWA Atsushi

ISEKI Kunitoshi

ISHIBASHI Hiromi

IsHIBASHI Toshiyuki

IsHiDA Tadao

IsHIDA Tadashi

IsHIDA Takashi

ISHIDA Yoji

IsHIGATSUBO Yoshiaki

IsHIGURO Atsushi

IsHIHARA Takashi

ISHIKAWA San-e

IsHIKAWA Takashi

IsHIKAWA Toru

IsHIMURA Eiji

ISHIZAKI Takeshi

IsHizukA Tatsuo

IsoBE Takeshi

IsoBe Yasushi

ISOZAKI Osamu

ITO Akira

ITo Chikako
ITo Daisuke

ITO Hiroshi

ITO Isao

ITO Satoru

ITO Satoshi

ITo Takafumi

ITo Takahito

ITO Tetsuhide

ITO Yasuhiko

ITOH Jugoh

ITOH Mitsuyasu

ITOH Tomonori

ITOH Yoshiaki

ITOI Takao

ITSUBO Mariko

IWAKIRI Katsuhiko

IWAKIRI Ryuichi

IWAKURA Hiroshi

IwAKURA Katsuomi

IwAMOTo Masahiro

IWANAGA Takashi

IwANO Masayuki

IWAO Yasushi

IWASAKI Hiromichi

IWASAKI Yasumasa

IwASE Masanori

IwATA Kentaro

IZUTSU Koji

JP Kraehenbühl

JoH Takashi

KABURAKI Jyunichi

KADOTA Jun-ichi

KAGAWA Tatehiro

KAITANI Kazuaki

KaJi Hidesuke

KAJI Hiroshi

KajIYAma Hiroshi

KAKEI Masafumi

KAKU Shin

KAMADA Arisu

Kamata Kouju

KAMEDA Hideto

KAMEI Satoshi

KAMEOKA Junichi

KAMIjYo Keiichi

KAMISAWA Terumi

KAMOI kyuzi

KAMOUCHI Masahiro

KANAI Takanori

KANAMOTO Naotetsu

Kanatsuka Azuma

KANAZAWA Ippei

KANDA Fumio

KANDA Hiroko

KANDA Takashi

KANDA Yoshinobu

KANEKO Hiroshi

KANEKO Takeshi

KAO Jia-Horng

KARASAWA Masamitsu

KARIO Kazuomi

KASAHARA Kei

KASAI Kikuo

KASAI Masaharu

KASAI Takatoshi

KASAYAMA Soji

KASHIDA Hiroshi

Katabami Takuyuki
KATAKAMI Naoto

KATAOKA Hiromi

Katayama Kazuhiro

KATAYAMA Naoyuki

KATO Junji

KATO Masahiko

KATO Mototsugu

KATSURA Hideki

KAWA Shigeyuki

KAWABATA Hiroshi

KAWABATA Yoshinori

KAWADA Norifumi

KAWAGUCHI Kazunori

KAWAGUCHI Tatsuya

KAWAHITO Yutaka

KAWAI Hiroshi

KAWAI Hiroya

KAWAI Shinichi

KAWAI Toshihide

KAWAKAMI Atsushi

KAWAKAMI Hiroshi

KAWAMURA Mitsuru

KAWANA Masatoshi

KAWANO Hiroaki

KAWANO Mitsuhiro

KAWASAKI Eiji

KAWASUGI Kazuo

KAWAYAMA Tomotaka

KEICHO Naoto

KICHIKAWA Kimihiko

KIDA Kozui

KIHARA Yasuki

KIKUCHI Eiki

KIKUCHI Hirotoshi

KIKUCHI Ken

KIKUCHI Kentaro

KIKUYAMA Masataka

KIM Haeryoung

KIMURA Genjiro

KIMURA Kazumi

KIMURA Kazuo

KIMURA Satoshi

KIMURA Shinya

KInjo Fukunori

KINOSHITA Ichiro

KinOSHITA Tomohiro

KInOshita Yoshikazu

KIRA Jun-ichi

KISO Shinichi

KITA Hiroto

Kitagawa Kazuo

KITAGAWA Yasuhisa

KitamURA Kazuya

KitamURA Kenichiro

KiTANAKA Akira

KiTANo Kiyoshi

KIYOI Hitoshi

KiYomoto Hideyasu

KIZAKI Masahiro

KOARAI Akira

KoBASHI Yoshihiro

KOBAYASHI Masaki

KoBAYASH Nobuyuki

KOBAYASHI Shigeto

KoHARA Katsuhiko

KoHGO Yutaka

KoHNO Nobuoki

KOHROGI Hirotsugu

KoIDE Yuji

KOIKE Ryuji
KoIzumi Tomonobu

KOKAME Koichi

KoKUBo Yasuaki

Komagata Yoshinori

KOMAI Norio

Komatsu H

KOMATSU Norio

Komatsu Sei

Komatsu Takashi

Komatsuda Atsushi

KOMORI Tetsuo

KOMORI Tsuyoshi

KONDO Fukuo

KonDO Kunikazu

KONDO Masashi

KonNo Satoshi

KONTA Tsuneo

KonYA Hiroyuki

KOTAKE Shigeru

KoukI Tsuyoshi

KoYA Daisuke

KOYAMA T.

KUBO Hiroshi

Kubota Ken

KUBOTA Ryuji

Kubota Sumihisa

Kubota Tetsuya

Kumada Takashi

KuMAGaI Tomohiro

Kumamoto Toshihide

Kunimoto Masanari

Kunishima Hiroyuki

KURASAWA Kazuhiro

KuRATA Takayasu

KURITA Takashi

KURIYAMA Kazutaka

KURODA Takeshi

KuROIwA Takashi

KUROIWA Yoshiyuki

KuROKAwa Mineo

KUROSAKI Masayuki

KuRosawa Hajime

KuROSE Takeshi

Kusama Yoshiki

Kusano Eiji

KUSANO Kengo

KusAno Motoyasu

Kusumoto Shigeru

KUSUNOKI Hiroaki

KusunOKI Susumu

KuwABARA Satoshi

KUWAHARA Koichiro

KUWAHIRA Ichiro

KUwANA Masataka

Kuwano Kazuyoshi

Matsuda Masafumi

MABE Katsuhiro

MAEGAWA Hiroshi

MAEMONDO Masaru

MAESAKI Shigefumi

MAESHIMA Akito

MAESHIMA Youhei

MAKINO Shigeki

MARUYAMA Haruhiko

MARUYAMA Hiroki

MARUYAMA Schoichi

Maruyama Taro

MASAKI Tsutomu

MASAKI Yasufumi 
MASSANi Marco

MASUDA Izuru

MASUYAma Tohru

MATSUBARA Mitsunobu

MATSUBARA Shiro

MATSUDA Akira

MATSUdA Hiroshi

MATSUDA Masayuki

MATSUDA Naoyuki

MATSUe Kosei

Matsui Makoto

MATSUI Toshimitsu

Matsukawa Yoshihiro

MATSUKI Michihiro

Matsumoto Hideyuki

Matsumoto Jun

Matsumoto Masanori

Matsumoto Michiaki

Matsumoto Nobuhiro

MAtsumoto Takayuki

MATSUMURA Itaru

Matsumura Masami

Matsunaga Kazuo

Matsunaga Kazuto

MATSUnO Yoshihiro

MATSUO Kunihiro

Matsuse Hiroto

Matsushita Shuzo

MATSUURA Bunzo

MATsuZAKI Yasushi

Migita Kiyoshi

Mimura Toshihide

Minakata Yoshiaki

Minamino Tetsuo

Minami Masahito

Minatoguchi Shinya

Mine Tetsuya

Minematsu Kazuo

MISU Tatsuro

Mitamura Hideo

Mitsui Jun

MitsutAke Kotaro

MiURA Takeshi

MiURA Tetsuji

Miwa Hiroto

MiYajIMA Hiroaki

MiYAKAWA Yoshitaka

MiYAKE Fumihiko

MIYAMORI Isamu

Mryamoto Kenji

MiY AMURA Nobuhiro

MiYASHITA Kotaro

MiYASHITA Naoyuki

MIYASHITATaiichiro

MiYata Masayuki

MIYAZAKI Shigeru

MIYAZAKI Shunichi

MIYAZAKI Yasushi

MiYAZAKI Yoshitsugu

MIYAZAWA Naoki

MiYAZAWA Teruomi

Miyoshi E.

Mizuki Masao

MizUKOSHI Eishiro

Mochida Satoshi

MochIZUKI Hitoshi

MochizUki Toshio

Momomura Shin-ichi

Momoshima Hirotaka

Monden Tsuyoshi
MonKawa Toshiaki

MORI Kiyoshi

MoRI Takefumi

MORI Takehiko

MoRIMOTO Konosuke

MORISE Zenichi

Morishit A Eriko

MORITA Hiroyuki

MORIWAKI Hisataka

MORIYA Tatsumi

MoToji Toshiko

Motoo Yoshiharu

Motoya Satoshi

MukaE Hiroshi

Mukoyama Masashi

Munakata Mitsuru

Mune Tomoatsu

MuRAKAMI Hirokazu

MURAKAMI Ichiro

MuraKami Kazunari

MuRAse Takashi

Murata Miho

Murata Mitsuru

Murayama Shigeo

Muso Eri

NAGAFUCHI Seiho

NAGAFujI Koji

NAGAI Hirokazu

NAGAI Katsura

NAGAI Sonoko

NAGAI Yayoi

NAGAO Takehiko

NAGASAKA Shoichiro

NAGASAWA Kohei

NAGASAWA Yasuyuki

NAGASHIMA Takao

NAGATA Eiichiro

NAGATA Ken

NAGATAKE Tsuyoshi

NAGOSHI Sumiko

NAITO Yuji

NAKABAYASHI Kimimasa

NAKAGAMI Tomoko

NAKAGAWA Masanori

NAKAGAWA Yasuaki

NAKAGAWA Yoshihisa

NAKAGAWA Yusuke

NAKAJIMA Kenichi

NAKAJIMA Takahiko

NAKAJIMA Tamio

NAKAJIMA atsushi

NAKAKUMA Hideki

NAKAMURA Hidetoshi

NAKAMURA Hirotoshi

NAKAMURA Jiro

NAKAMURA Kazuhiko

NAKAMURA Masato

NAKAMURA Minoru

NAKAMURA Naoto

NAKAMURA Shinichi

NAKAMURA Tadashi

NAKAMURA Tsukasa

NAKAMURA-UCHIYAMACZ

Fukumi

NAKANISHI Norifumi

NAKANISHI Yoichi

NAKANO Imaharu

NAKANO Takashi

NAKANO Yasutaka
NAKAO Toshiyuki

NAKASE Hiroshi

NAKASEKO Chiaki

NAKASHIMA Hitoshi

NAKATA Koh

NAKATANi-Enomoto Setsu

NAKAYA Izaya

NAKAYAMA Katsutoshi

NAKAYAMA Masaaki

NAKAZATO Masamitsu

NAKAZATO Yoichi

NAKAZATO Yuji

NANKI Toshihiro

NARA Masayuki

NARITA Takuma

NARITOMI Hiroaki

NARUMI Shunji

NIIMI Akio

NiITSU Nozomi

NINOMIYA Toshiharu

NISHI Masahiro

NISHI Sinichi

NISHIDA Makoto

NiSHIKAWA Hiroaki

NISHIKAWA Masanori

NISHIKORI Momoko

NISHIMORI Isao

NISHIMURA Genichi

NISHIMURA Haruo

NishimURA Kazunobu

NISHIMURA Shigeyuki

NISHIMURA Yoshihiro

NISHINO Ichizo

NISHINO Takayoshi

NISHIOKA Yasuhiko

NishiY AMA Kazutoshi

NitoBe Joji

NiTTA Kosaku

NoDA Keita

NODA Takahiro

NoDA Yutaka

NoGAwA Shigeru

NomUra Masatoshi

NomURa Shosaku

NONAKA Ikuya

Notsumata Kazuo

NozAKI Hiroyuki

NOZAKI Naoki

OBA Shino

OBARA Katsutoshi

ODA Hirotaka

ODA Koji

OGA T.

OgaHARA Satoru

Ogata Atsushi

OGAWA Hiromasa

OGAWA Hisao

Ogawa Susumu

OGAWA Wataru

OGINO Mieko

OHASHI Haruhiko

OHASHI Hiroshige

OHE Yuichiro

OHIRA Hiromasa

OHMAGARI Norio

OHNISHI Hirohide

OHNISHI Kazunori

OHNISHI Kenji

OHNO Hiroshi
OHNO Hitoshi

OHNO Iwao

OHNo Yasuhiro

OHNO Yoichi

OHSAKI Yoshinobu

Oнта Kazuhide

OHTA Masayuki

OHTE Nobuyuki

OHYA Yasushi

OHYANAGI Mitsumasa

OIDA Koji

OIKAWA Shinichi

OIKAWA Yoichi

OIsHI Kazunori

Oiso Yutaka

OIzUMI Satoshi

OKA Mikio

OkA Teruaki

OKADA Hiroyuki

OKADA Jun

OKADA Sadanori

OKADA Shuichi

OKADA Yosuke

OKAMOTO Hiroaki

OKAMOTO Kohichiro

OKaмото Koichi

OKAMOTO Masataka

OKAмото Shinichiro

OKAMURA Takashi

OKAYAMA Akihiko

OKAYAMA Naotsuka

OKAYAMA Satoshi

OKAZAKI Kazuichi

OKAZAKI Ryo

OKI Yutaka

OKIMOTo Tadayoshi

OKIMURA Yasuhiko

OKUMA Yasuyuki

OKUMURA Ataru

OKUMURA Ken

OKUMURA Kenji

OKUMURA Tetsu

OKURA Yuji

OMOTO Eijiro

OMURA Masao

ONJi Morikazu

OnODERA Osamu

OnOUE Kenji

ONUMA Tomio

OSANAI Kazuhiro

OSANAI Shinobu

OTAKA Michiro

OTsuji Yutaka

OZAKI Shuji

Ozawa Toshifumi

Ozono Keiichi

RAKUGI Hiromi

RYUZAKI Munekazu

SAIKAWA Tetsunori

SUGISAKI Katsunori

SADJJADI Seyed Mahmoud

SAGARA Hironori

SAHASHI Ko

SAIBARA Toshiji

SAIJO Yasuo

SAISHO Yoshifumi

SAITO Nobuhito

SAITO Takafumi 
SAITO Takao

SAKA Hideo

SAKAGUCHI Kazuhiko

SAKAI Akira

SAKAI Akito

SAKAI Fumikazu

SAKAKIBARA Hiroki

SAKAKIBARA-KONISHI Jun

SAKAMOTO Hisato

SAKAMOTO Susumu

SAKURA Hiroshi

SAKURAI Akihiro

SAKURAI Shigeru

SANADA Shoji

SANAKa Tsutomu

SASADA Shinji

SASAKI Takeshi

SASAKI Tamaki

SASAKI Yuka

SASATOMI Yoshie

SATA Makoto

SATA Michio

SATo Atsuhisa

SATo Hiroshi

SATo Keizo

SATo Kojiro

SATO Taro

SATO Yuzo

SATOH Katashi

SATOH Noriko

SATOH Tetsuro

SATOMI Kazuhiro

SAWABU Norio

SAWADA Shigemasa

SAWAKI Akira

SAYAMA Koichi

SEKI Toshihito

SETOGuCHI Yasuhiro

SEYAMA Kuniaki

SHIBA Nobuyuki

SHIBAGAKI Yugo

SHIBATA Yoko

SHIJUBo Noriharu

ShIKATA Kenichi

SHIma Yoshihito

SHIMABUKURO Michio

SHIMADA Akira

SHIMADA K.

SHIMADA Kazunori

SHIMADA Yutaka

SHIMANO Hitoshi

SHIMAZAKI Chihiro

SHIMAZU Akira

SHIMIZU Akihiko

SHIMIZU Akira

SHIMIZU Eiji

SHIMIZu Nobuyuki

SHIMIZU Wataru

SHIMIZU Yuichi

SHIMODA Kazuya

SHImojima Yasuhiro

SHIMOKAWA Hiroaki

SHIMOMURA Iichiro

SHIMONO Nobuyuki

Shimosawa Tatsuo

ShimosegaWA Tooru

SHINAGAWA Naofumi

SHINKAI Masaharu

SHINOTO Hitoshi

SHIOI Tetsuo
SHIOMI Toshiaki

SHIOTANI Akiko

SHIOYA Takanobu

SHIRAI Kohji

SHIRATORI Keiko

SHIRATORI Yoshimune

SHIsHido Tetsuro

SHOJI Mikio

SHoJi Tetsuo

SoBuE Gen

SoEJIMA Hirofumi

SONOO Masahiro

Suematsu Eiichi

SUGA K.

Suga Tatsuo

SugAI Tamotsu

SugawARA Akira

SugIE Hideo

SugIE Takumi

SuginARA Takashi

Sugimoto Toshiro

Sugimoto Toshitsugu

SugImURA Yoshihisa

SugIURA Yoshihiro

SugIYAMA Hitoshi

SugIYAMA Seigo

SugiYama Toshiro

Sumida Yasuhiro

Sumino Yasukiyo

SunAdA Yoshihide

SunAmI Kazutaka

Suwa Tetsuya

SuYAma Masafumi

SuZUKI Atsushi

SuZUKI Daisuke

SuZUKI Eiichi

Suzuki Fumitaka

SuzUKI Hidekazu

SUZUKI Hiromichi

SuzUKI Katsuhiro

Suzuki Kazuo

SuZUki Kazuyuki

SuZUKi Masaru

SuZUKI Ritsuro

SuZUKI Shigeaki

SuzUKI Takahiro

SuZukI Toru

Suzuki Tsuyoshi

Suzuki Yasuo

SuzUKI Yoshiki

SuZUMIY A Junji

SUZUMURA Akio

Tokimatsu Issei

TABEI Kaoru

TACHIBANA Masaaki

TADA Yoshifumi

TAGAmi Tetsuya

TAGUCH Isao

TAHARA Yoshihiro

TAJIRI Junichi

TAJIRI Yuji

TAKADA Minoru

TAKADA Toshinori

TAKAGI Atsushi

TAKAGI Hitoshi

TAKAGI Junko

TAKAGI Shigeharu

TAKAHASHI Kazuhisa

TAKAHASHI Kazuma
TAKAHASHI Kazushi

TAKAHASHI Naohiko

TAKAHASHI Naoto

TAKAHASHI Ryosuke

TAKAHASHI Shinichi

TAKAHASHI Tomosaburo

TAKAHASHI Yoshihisa

TAKAHASHI Yutaka

TAKAKURA Shunji

TAKAMI Akiyoshi

TAKANO Koji

TAKANO Toshimi

TAKAO Toshihiro

TAKASAKI Yoshinari

TAKASHIMA Hiroshi

TAKASHIMA Shutaro

TAKASU Nobuyuki

TAKATA Tohru

TAKATORI Hajime

TAKATsuka Naoyoshi

TAKAYAMa Koichi

TAKAYANAGI Ryoichi

TAKEDA Atsushi

TAKEDA Eiji

TAKEDA Hidetaka

TAKEDA Hiroaki

TAKEDA Yoshiyu

TAKEhara Tetsuro

TAKEI Izumi

TAKEMURA Genzou

TAKEISHI Yasuchika

TAKEMURA Tamiko

TAKENAKA Tsuneo

TAKENO Mitsuhiro

TAKEUCHI K.

TAKEUCHI Yasuhiro

TAKEYAMA Yoichi

TAKIGUCHI Yuichi

TAKIUCHI Hiroya

TAKIYAMA Yoshihisa

TAKUBO Takayuki

TAMAOKA Akira

TAMURA Kazuo

TAMURA Koichi

TAMURA Naohisa

TAMURA Naoto

TANABE Akiyo

TANABE Kengo

TANABE Nobuhiro

TANAHASHI Norio

TANAKA Eiji

TANAKA Eisaku

TANAKA Hiroshi

TANAKA Keiko

TANAKA Kiyoshi

TANAKA Masami

TANAKA Shinji

TANAKA Yasuhito

TANAKA Yoshiya

TANAKA Kortaro

TANI Kenzaburo

TANIGAWA Keiichiro

TANIGUCHI Atsuo

TANIGUCHI Masami

TANIGUCHI Shigeki

TANIWAKI Masafumi

TANIYAma Matsuo

TANIZAWA Yukio

TAOOKA Yasuyuki

TASAKA Sadatomo
Tateda Kazuhiro

TATSUNO Ichiro

TAUCHI Tetsuzo

Tazuma Susumu

Teragawa Hiroki

TERAI Chihiro

Terai Shuji

Terajima Kenshi

TERamoto $\mathrm{K}$

TERASAKI Shuichi

Terayama Yasuo

TERUI Yasuhito

TESHIMA Takanori

ToBIMATSU Shozo

TOHYAMA Kaoru

Tojo Arinobu

Tojo Katsuyoshi

TokUDA Haruhiko

TokUE Yutaka

TokUMO Hironori

ToKUnAGA Daisaku

ToKURA Takehiko

TOKUSHIGE Katsutoshi

Tominaga Kazunari

Tomita Naoto

Tomit A Naruya

TOMIY AMA Yoshiaki

Tomo Tadashi

TOMODA Fumihiro

ToshikUni Nobuyuki

TOYODA Kazunori

Toyonaga Tetsushi

Toyoshima Itaru

Tsubor Kumiko

TsuBoI Yoshio

TSUCHIYA Ken

TSUDA Hiroyuki

TsuDA Kinsuke

Tsuji Sadatoshi

TsujI Shoji

TSUJII Satoru

TsujINo Ichizo

Tsukamoto Kazuhisa

TsukijI Jun

Tsunada Seiji

TSURUYA Kazuhiko

TsuTsur Hiroyuki

UBARA Yoshibumi

UCHIDA Keiko

UCHIGATA Yasuko

UCHIYAMA Shinichiro

UEDA Seiji

UEDA Takanori

Ueki Kazue

UEKI Kojiro

UEMURA Masahito

UEMURA Shiro

UENO Takahumi

UENO Yoshiyuki

Ueshima Kenji

UGAWA Yoshikazu

UMEHARA Hisanori

URA Nobuyuki

URABE Takao

URANO Tetsuya

URATA Hidenori

URITA Yoshihisa

UsUI Joichi

UsUI Noriko 
USUI Soichiro

UsUI Takeshi

Usuki Kensuke

UTO Hirofumi

UTSUNOMIYA Yasunori

VAN DER MEER Jos W.

W ADA Hiroo

WADA Hideo

WADA Jun

WADA Masako

WAKABAYASHI Tokio

WAKAI Toshifumi

WAKAYAMA Yoshihiro

WAKINO Shu

WAKITA Atsushi

W AKUI Hideki

WAKUTANI Yosuke

WATADA Hirotaka

WATANABE Akira

WATANABE Hiroshi

WATANABE Hiroyuki

WATANABE Ichiro

Watanabe Kentaro

WatANABE Mamoru

WATANABE Sumio

Watanabe Tetsu

WATANABE Tsuyoshi

Watanabe Yoichi

Wu Bin
YAGI Kunimasa

YAKUSHIJ Yusuke

YAKUSHIJIN Yoshihiro

YAMADA Akira

YAMADA Chizumi

YAMADA Hidehiro

YAMADA Kentaro

YAMADA Masahito

YAMADA Yasuhide

YAMADA Yuichiro

YAMAGATA Kunihiro

YAMAGATA Mitsunori

YAMAGATA Toshiyuki

YAMAGISHI Shoichi

YAMAGUCHI Etsuro

YAMAGUCHI Hiroki

YAMAGUCHI Motoko

YAMAGUCHI Shuhei

YAMAGUCHI Toshiyuki

YAMAKITA Noriyoshi

YAMAMORI Ikuo

YAMAMOTO Haruko

YAMAMOTO Hiroyuki

YAMAMOTO Kazuhiro

YAMAMOTO Masahiro

YAMAMOTO Mayumi

YAMAMOTO Motohisa

YAMAMOTO Shojiro

YAMAMOTO Takeshi

YAMAMOTO Yasumasa

YAMAO Kenji

YAMASHINA Shohei
YAMASHIRO Kazuo

YAMASHITA Takeshi

YAMAUCHI Kohei

YAMAUCHI Toshimasa

YAMAUCHI-TAKIHARA Keiko

YAMAYA Mutsuo

YAMAZAKI Jun-ichi

YAMAZAKI Masahide

YAMAZAKI Yasushi

YANAGAWA Tatsuo

YANAGIHARA Katsunori

YANASE Toshihiko

YANO Hideki

YANO Seiji

YAO Takashi

YASAKA Masahiro

YASODA Akihiro

YASUDA Hideo

YASUDA Hitoshi

YASUDA Kazuki

YASUDA Yoshinari

YASUE Hirofumi

YASUHIRO Akai

YASUI Masahide

YASUKAWA Masaki

YASUOKA Akira

YASUYUKI Nasuhara

YATOMI Yutaka

YATSUHASHI Hiroshi

YAZAKI Masahide

YoKOCHI Fusako

YOKOKAWA Hirohide
YOKOYAMA Akihito

YOKOYAMA Keitaro

YonedA Masashi

YONEDA Masato

YosHIDA Akio

YosHIDA Haruhiko

YosHIDA Katsunori

YosHIDA Koichiro

YOSHIDA Masaharu

YosHIDA Masaki

YoshIDA Masanori

YoshidA Minoru

YosHIDA Shunji

YoshidA Toshihide

YoshiI Fumihito

Yoshikawa Masahide

YoshIKAWA Toshikazu

YosHIKAWA Tsutomu

YOSHIMATSU Hironobu

YOSHIMURA Ashio

YoshIMURA Hiroshi

YoshIMURA Michihiro

YosHIOKA Kentaro

YoshiY AMA Minoru

YoshIYAMA Takashi

YOTSUYANAGI Hiroshi

YuDA Satoshi

YUMURA Wako

YuZAWA Yukio

ZENIYA Mikio 